Guedes, DCV, Brito, SA \& Silva, DR. (2020). The importance of pharmaceutical care in women during pregnancy. Research, Society and Development, 9(7): 1-20, e714974626.

\title{
A importância do cuidado farmacêutico em mulheres no período gestacional
}

The importance of pharmaceutical care in women during pregnancy

\section{La importancia de la atención farmacéutica en las mujeres en el período de gestión}

Recebido: 17/05/2020 | Revisado: 20/05/2020 | Aceito: 25/05/2020 | Publicado: 07/06/2020

\section{Damiris de Carvalho Vieira Guedes}

ORCID: https://orcid.org/0000-0003-2720-2633 Programa de pós-graduação da Faculdade Santa Maria, Brasil

E-mail: damiresigt@hotmail.com

Samara Alves Brito

ORCID: https://orcid.org/ 0000-0002-2808-7836

Faculdade Santa Maria, Brasil

E-mail: samaralvesbritobrito19@gmail.com

Danielle Rocha Silva

ORCID: https://orcid.org/0000-0002-2949-2008

Faculdade Santa Maria, Brasil

E-mail: prof.daniellerocha@hotmail.com

\section{Resumo}

A atenção farmacêutica é um serviço relevante na promoção do uso racional de medicamentos e sucesso farmacoterapêutico, devido ao conhecimento e acessibilidade existente por parte do farmacêutico. Logo, denota-se grande importância da prestação desse serviço às gestantes, em razão dos possíveis riscos materno-fetais associados ao medicamento. Bem como a doenças pré-existentes e condições durante este período que requerem a medicalização, como hipertensão arterial e diabetes mellitus gestacional, que apresentam grande relevância na saúde pública brasileira, em virtude da incidência que exibem e das complicações que podem provocar, consistindo em cenários oportunos para o acompanhamento farmacêutico visando auxiliar a adesão ao tratamento e promoção de saúde. Nesse contexto, objetivou-se compreender a importância do farmacêutico clínico na orientação farmacológica durante a gestação. Foi realizada uma pesquisa nas bases de dados Scielo, PubMed, Google Scholar e 
ScienceDirect, usando os descritores: categorias de risco; hipertensão; diabetes mellitus; automedicação; gravidez; náuseas; vômitos; eclampsia e assistência farmacêutica, todos selecionados dos DeCS nos idiomas: Português e Inglês. Evidenciou-se nesse estudo, a importância da atenção farmacêutica na gravidez, relacionado à orientação segura e adequada de terapias medicamentosas, reduzindo a automedicação, como também no processo de adesão terapêutica, através do acompanhamento contínuo e regular, destacando a importância da realização correta do tratamento e sugerindo intervenções terapêuticas necessárias, contribuindo assim para a melhora da qualidade de vida materno-fetal. Portanto, essa pesquisa assume relevância ímpar, uma vez que explanou a atenção farmacêutica para gestantes, colaborando para o desenvolvimento de ações e campanhas visando mostrar e conscientizar a importância do farmacêutico clínico durante a gestação.

Palavras-chave: Assistência farmacêutica; Gravidez; Uso de medicamentos.

\section{Abstract}

Pharmaceutical care is a relevant service to promote the rational drug use and pharmacotherapeutic success, due to the pharmacist's knowledge and accessibility. On this regard, there is a great relevance on providing this service to pregnant women, due to the possible maternal-fetal risks associated with some drugs. As well as pre-existing diseases and conditions for instance, arterial hypertension and gestational diabetes mellitus which require the use of pharmacological therapies and are of great relevance in Brazilian public health. Due to the high incidence and the complications they may cause, it is an opportune period for the pharmaceutical care to assist treatment adherence and consequently achieve therapeutic success. In this context, the aim of this study is to understand the importance of clinical pharmacists in the pharmacological care during pregnancy. A research was carried out in the databases Scielo, Google Scholar, PubMed and ScienceDirect databases, using the descriptors: risk categories; hypertension; diabetes mellitus; self-medication; pregnancy; nausea; vomiting; eclampsia and pharmaceutical assistance, all selected from DeCS in the languages: Portuguese and English. The importance of pharmaceutical care in pregnancy was evidenced through this study regarding not only the safety and adequate guidance to the use of drug therapies to reduce self-medication, but also the therapeutic adherence process through continuous and regular monitoring. Hence, contributing to the improvement of both maternal and fetal life quality by the pharmaceutical care that highlights whenever necessary the importance of correct treatment compliance and advises the need of therapeutic interventions. Therefore, this study has a unique relevance once it explained the 
pharmaceutical care importance for pregnant women. Furthermore, it collaborates for the development of plans and actions that aim to expose and conscientize the importance of the clinical pharmacist during this period.

Keywords: Pharmaceutical services; Pregnancy; Drug utilization.

\section{Resumen}

La atención farmacéutica es un servicio relevante en la promoción del uso racional de medicamentos y el éxito farmacoterapéutico, debido al conocimiento y accesibilidad del farmacéutico. Así pues, su gran importancia en la oferta de estos servicios hacia las mujeres embarazadas, en razón de la probabilidad de los riesgos materno-fetal adjuntados al medicamento. Bien como las enfermedades pré-existentes y las condiciones durante este período que requieren la medicalización, como presión arterial alta y diabetes mellitus gestacional, que presentan gran relevancia en la salud pública brasileña, en virtud da la ocurrencia exhibidas y de las complicaciones que pueden provocar, constituyendo cuadros oportunos para el acompañamiento farmacéutico visando auxiliar la adhesión hacia a los procedimiento y promoción de la salud. En este contexto, el objetivo fue comprender la importancia del farmacéutico clínico en la orientación farmacológica durante el periodo de la gestación. Fue realizada una investigación en las bases de datos Scielo, Google Académico, PubMed y ScienceDirect, usando los descriptores: categorías de riesgo; hipertensión; diabetes mellitus; automedicación; embarazo; náusea; vómitos; eclampsia y asistencia farmacéutica, todos seleccionados de los DeCS y los idiomas: portugués e inglés. Fue evidente en este estudio, la importancia de la atención farmacéutica en el embarazo, relacionado hacia la orientación segura y adecuada de las terapias farmacológicas, la reducción de la automedicación, así como en el proceso de adherencia terapéutica, a través del monitoreo y regulación continua, recalcando la importancia de la realización correcta del tratamiento y apuntando intervenciones terapéuticas necesarias, contribuyendo de esta forma para la mejora de la calidad de vida materno-fetal. Por tanto, esta investigación se pone en relevancia singular, una vez que explica la atención farmacéutica para mujeres embarazadas, ayudando en el desarrollo de acciones y campañas con el fin de demostrar y sensibilizar sobre la importancia del farmacéutico clínico durante el embarazo.

Palabras-claves: Servicios farmacéuticos; Embarazo; Utilización de medicamentos. 


\section{Introdução}

A gestação representa um importante período na vida da mulher, tanto na esfera emocional refletida no fato de gerar uma nova vida e suas responsabilidades correspondentes, como também na esfera física devido às várias modificações experimentadas pelo organismo da mãe, provocando um aumento na vulnerabilidade imunológica e demanda nutricional, que, uma vez não considerados adequadamente, podem atrapalhar o desenvolvimento fetal, bem como o bem-estar materno (Souza \& Barros, 2017).

Porquanto, estas modificações físicas, somadas ao elevado índice de doenças crônicas entre mulheres, retratam um cenário de maior cautela por destacarem a necessidade da utilização constante de medicamentos e até mesmo de uma maior quantidade e variedade, tornando o acompanhamento clínico fundamental para o uso eficaz e principalmente seguro dos fármacos (Silva \& Marques, 2019).

Uma vez que a maioria destes possui a capacidade de permear a barreira placentária, podendo provocar efeitos adversos no feto em evolução, os quais geralmente são tóxicos. Destacando-se, a extrema importância de avaliar o risco-benefício relacionado ao uso da terapia medicamentosa (Andrade, 2019).

O elevado consumo de medicamentos industrializados por parte da população brasileira tem favorecido a familiaridade com essas substâncias e impulsionado o uso indiscriminado e/ou automedicação, sendo esta prevalente entre o sexo feminino e significativamente preocupante entre as gestantes, pela falta de conhecimento específico destas sobre as reações adversas e os princípios ativos no tocante à medicação (Delgado \& Vriesmann, 2018). A polifarmácia é uma prática muito frequente na população atendida nos serviços da atenção primária do Sistema Único de Saúde (SUS), seja pela prescrição demasiada ou pela automedicação, podendo estar relacionada ao uso indiscriminado ou inapropriado de medicamentos (Nascimento, et al., 2017).

Associado aos fatores supracitados, o profissional farmacêutico tem papel fundamental no processo, já que detém o conhecimento específico dos medicamentos, podendo auxiliar a adesão ao tratamento do paciente e contribuir para a avaliação do risco-benefício da terapia medicamentosa. Soma-se a isto que durante a gestação, a farmácia clínica promove a segurança em relação ao potencial efeito tóxico associado ao uso de medicamentos, e fornece educação em saúde à gestante no tratamento prescrito (Burkey \& Holmes, 2013).

Tendo em vista estas considerações sobre o tema, o presente trabalho tem como objetivo compreender a importância do farmacêutico clínico na orientação farmacológica 
durante a gestação. Como o profissional é de fácil acesso e que dispõe do conhecimento singular dos fármacos, que, nesse período, mostra-se crucial, devido às inúmeras complicações que podem ocorrer através do consumo dessas substâncias e da necessidade de suplementação para suprir a exigência nutricional do feto.

\section{Metodologia}

A pesquisa foi realizada através de uma revisão narrativa da literatura no período de fevereiro a maio de 2020, com ênfase na pesquisa de artigos em língua portuguesa e inglesa, indexados nas bases de dados eletrônicas Scientific Electrinic Libray Online (SCIELO), Google Scholar, PubMed e ScienceDirect, compreendendo o intervalo temporal de 2010 a 2020.

Para a efetivação da pesquisa foram utilizados os descritores: "categorias de risco"; "hipertensão"; "diabetes mellitus"; "náuseas e vômitos"; "automedicação"; "gravidez"; "assistência farmacêutica", em uma busca ativa livre. Em adição, foram selecionados e empregados os filtros, além da análise dos títulos e resumos dos artigos que permaneceram após a aplicação dos filtros, na qual foram descartados os artigos múltiplos entre as bases de dados e aqueles que divergiam da temática da pesquisa. Os descritores usados neste estudo estão devidamente cadastrados nos Descritores em Ciências da Saúde (DeCS).

Visando tornar a pesquisa mais robusta, foram determinados os seguintes critérios de inclusão: a) artigos publicados nos últimos 10 anos; c) artigos completos de acesso aberto; d) artigos nos idiomas inglês e português. Assim como, foram definidos também critérios de exclusão, como: a) estudos em duplicidade nas as bases de dados consultadas; b) estudos não divergentes ao tema. Por fim, foram selecionados os artigos de maior relevância de acordo com o tema para a elaboração desta revisão visando analisar e discutir o que existe de pertinente sobre o tema na literatura científica.

\section{Resultados e Discussão}

Realizando a busca livre de artigos sobre o tema, sem o emprego dos critérios de inclusão e dos critérios de exclusão, foram identificados um total 2.195 .904 publicações, destas foram utilizadas 45 publicações, após a aplicação dos critérios de inclusão e exclusão, conforme a Tabela 1 . 
Tabela 1-Método de busca nas bases de dados SciELO, PubMed, Google Scholar e ScienceDirect.

\begin{tabular}{|c|c|c|}
\hline DESCRITORES & $\begin{array}{c}\mathbf{N}^{\circ} \text { DE ARTIGOS } \\
\text { ENCONTRADOS POR } \\
\text { BASE DE DADOS }\end{array}$ & $\begin{array}{c}\mathbf{N}^{\circ} \text { DE ARTIGOS } \\
\text { UTILIZADOS POR } \\
\text { BASE DE DADOS }\end{array}$ \\
\hline 1) Categorias de risco & 52.100 (Google Scholar) & 3 (Google Scholar) \\
\hline \multirow{3}{*}{ 2) Hipertensão } & 374 (Scielo) & 3 (Scielo) \\
\hline & 359.614 (ScienceDirect) & 1 (ScienceDirect) \\
\hline & 65.300 (Google Scholar) & 4 (Google Scholar) \\
\hline \multirow[t]{2}{*}{ 3) Diabetes mellitus } & 1.170 .000 (Google Scholar) & 7 (Google Scholar) \\
\hline & $\begin{array}{l}197.244 \\
\quad \text { (ScienceDirect) }\end{array}$ & 2 (ScienceDirect) \\
\hline \multirow[t]{2}{*}{ 4) Automedicação } & 13.300 (Google Scholar) & 3 (Google Scholar) \\
\hline & 55 (PubMed) & 2 (PubMed) \\
\hline \multirow[t]{2}{*}{ 5) Gravidez } & 238.917 (ScienceDirect) & 3 (ScienceDirect) \\
\hline & 46.900 (Google Scholar) & 6 (Google Scholar) \\
\hline 6) Assistência farmacêutica & 15.100 (Google Scholar) & 2 (Google Scholar) \\
\hline 7) Náuseas e vômitos & 15.200 (Google Scholar) & 3 (Google Scholar) \\
\hline \multirow[t]{2}{*}{ 8) Eclâmpsia } & 21.800 (Google Scholar) & 6 (Google Scholar) \\
\hline & $\begin{array}{c}\text { TOTAL = 2.195.904 } \\
\text { publicações }\end{array}$ & TOTAL $=45$ publicações \\
\hline
\end{tabular}

Fonte: Autores.

\subsection{Categorias de risco}

A tragédia da talidomida tornou-se um marco histórico da farmacovigilância. A prescrição desse medicamento para grávidas com queixas de náuseas e vômitos no primeiro trimestre da gestação teve início no fim dos anos 50 e, na década posterior, confirmaram-se as malformações congênitas geradas por sua utilização, com ênfase na interrupção do desenvolvimento das extremidades nos embriões e nas más formações de órgãos internos, como o coração (Moro \& Invernizze, 2017). 
Até o fim da década de 40, presumia-se que o embrião e o feto estavam protegidos de agentes externos. Todavia, a partir de 1941, pesquisas revelaram que os medicamentos teriam a capacidade de ocasionar malformações congênitas, dado que a barreira placentária não inibe a passagem de todas as drogas, mas ocorre uma seleção conforme suas características (Nascimento et al., 2016). Porém, as informações científicas a respeito do real risco relacionado a utilização de medicamentos durante a gestação são poucas (Menezes, et al., 2014; Stock \& Norman, 2019).

Neste sentido, com o intuito de direcionar o prescritor na seleção da terapia mais apropriada para as gestantes, a agência americana Food and Drug Administration - FDA classificou os fármacos em relação aos efeitos que promovem na gravidez em categorias de risco, como descrito na Tabela 2 (Van Gelder; Jong-Van den Berg \& Roeleveld, 2014).

Tabela 2- Classificação de risco dos fármacos utilizados no período gestacional de acordo com a Food and Drug Administration - FDA.

\section{CATEGORIA}

DESCRIÇÃO

\begin{tabular}{c|c}
\hline Categoria de risco A & $\begin{array}{c}\text { Nos estudos controlados em mulheres grávidas, não apresentaram } \\
\text { risco fetal no primeiro trimestre de gravidez. E por sua vez, sem } \\
\text { evidências de risco nos demais trimestres da gestação, sendo } \\
\text { remota a possibilidade de dano fetal. }\end{array}$ \\
\hline Categoria de risco B & $\begin{array}{c}\text { Nos estudos em animais, não apresentaram risco fetal, porém não } \\
\text { há registro de estudos controlados em gestantes, ou então, os } \\
\text { estudos em animais apresentaram riscos, mas que não foram } \\
\text { confirmados em estudos controlados em mulheres grávidas. }\end{array}$ \\
\hline Categoria de risco C & $\begin{array}{r}\text { Ausência de estudos em animais e em mulheres grávidas, ou } \\
\text { então, os estudos em in vivo apresentaram risco. }\end{array}$ \\
\hline Categoria de risco D & $\begin{array}{r}\text { Apresentaram evidências positivas de risco fetal humano, no } \\
\text { entanto, os benefícios potenciais para a mulher podem, } \\
\text { eventualmente, justificar o risco. }\end{array}$ \\
\hline Categoria de risco X & $\begin{array}{r}\text { Nos estudos em animais e gestantes, acarretaram anomalias fetais, } \\
\text { havendo evidência de risco para o feto que se sobrepõe ao possível } \\
\text { efeito benéfico para a paciente. }\end{array}$ \\
\hline
\end{tabular}

Fonte: Adaptado de ANVISA, 2010.

Entretanto, para uma considerável fração de fármacos, não existem informações científicas a respeito do real risco relacionado a sua utilização durante a gestação o que 
impossibilita a categorização do mesmo de acordo com o FDA. Além disso, algumas gestantes apresentam comportamento de risco ao realizar a automedicação ou o uso irracional de medicamentos (Menezes et al., 2014). Diante disso, em 2015 a FDA divulgou a "Regra de rotulagem para gravidez e lactação" (PLLR), que consiste em uma determinação que facilita a divulgação clara de informações a respeito dos riscos associados ao uso de fármacos durante a gravidez, uma vez que as categorias de risco até então utilizadas são frequentemente mal interpretadas e muito simplista, não informando efetivamente o risco que um medicamento pode conter para gestantes. Esta determinação estabelece modelos de como as informações sobre o uso de medicamentos durante a gravidez e a amamentação devem ser exibidas nos rótulos e bulas dos medicamentos e produtos biológicos.

\subsection{Hipertensão arterial na gestação}

Durante a gestação, algumas mulheres são acometidas pela hipertensão arterial, a qual consiste em uma doença do sistema cardiovascular provocada por vários fatores, que promovem a alteração dos níveis da pressão arterial, podendo motivar a prematuridade do feto e baixo peso no nascimento, bem como colocando em risco a saúde do binômio materno-fetal (Prado, et al., 2017).

Dentre os múltiplos fatores que influenciam o seu surgimento, encontram-se a situação nutricional, histórico de pré-eclâmpsia, histórico familiar, obesidade e diabetes mellitus, destacando a predisposição existente entre as mulheres com idade nos extremos da fase reprodutiva (Moura, 2010).

Esta síndrome hipertensiva possui grande relevância na saúde pública brasileira, uma vez que é considerada como a maior causa de morbimortalidade materna e perinatal, representando cerca de $25 \%$ dos óbitos maternos investigados. Demonstrando a necessidade do acompanhamento da pressão arterial e do seguimento farmacoterapêutico para reduzir as complicações decorrentes dessa síndrome e aumentar o sucesso da terapia (Melo, et al., 2015).

De acordo com as Diretrizes Brasileiras de Hipertensão publicadas em 2010, existem alguns tipos de hipertensão arterial na gestação, sendo classificados de acordo com o período em que ocorre e as características que apresenta (Brasil, 2010). A Tabela 3 descreve as classes de categorização da hipertensão arterial na gestação. 
Tabela 3- As classes da hipertensão arterial na gestação.

CLASSE

\begin{tabular}{l}
\hline Pré-eclâmpsia \\
\hline Eclampsia \\
\hline Hipertensão crônica \\
Pré-eclâmpsia superposta \\
à hipertensão crônica
\end{tabular}

\section{Hipertensão gestacional}

Manifesta-se após a vigésima semana de gestação, sendo acompanhada de proteinúria.

Condiz à pré-eclâmpsia complicada por convulsões que não podem ser associadas a outros motivos.

Revela-se antes da gestação, no período que antecede a vigésima semana de gravidez ou doze semanas após o parto. Consiste no aumento agudo da pressão arterial associado a proteinúria, trombocitopenia ou alterações da função hepática, em gestantes que excederam a vigésima semana de gestação e que apresentam hipertensão crônica.

Caracteriza-se por hipertensão arterial identificada após a vigésima semana de gestação, com ausência de proteinúria.

Fonte: Adaptado de Brasil, 2010.

O mecanismo fisiopatológico da pré-eclâmpsia mais adotado na atualidade é uma máadaptação placentária, em que há um aumento da reatividade e permeabilidade vascular e ativação da coagulação com danos principalmente para o endotélio vascular, os rins, sistema nervoso central, fígado e placenta, prejudicando o funcionamento adequado deste último por conta da diminuição do aporte sanguíneo (Kahhale; Francisco \& Zugaib, 2018).

Por este motivo, a utilização de baixas doses de ácido acetilsalicílico iniciada na $12^{\mathrm{a}}$ à $14^{\text {a }}$ semana de gestação nas grávidas que apresentam risco moderado e elevado de préeclâmpsia pode ser benéfica (Ferreira, et al., 2017). Este cenário também se aplica à suplementação de cálcio, onde a ingestão adequada ou superior às recomendações dietéticas diárias exibiram uma inferior sensibilidade ao sódio e baixa prevalência de pré-eclâmpsia (Silva, et al., 2011).

O tratamento da síndrome hipertensiva na gestação abrange ações não farmacológicas, como dieta adequada, diminuição do consumo de substâncias que contenham cafeína, redução da prática de atividades físicas, abandono de hábitos não saudáveis como a ingesta de álcool e consumo de cigarro (Vettore, et al., 2011). Bem como ação farmacológico, que corresponde ao uso de medicamentos anti-hipertensivos, os quais são administrados quando a pressão arterial se encontra acima de 150/100 mmHg. Em grávidas com pré-eclâmpsia, que possuem o quadro clínico estabilizado sem necessidade de parto urgente, está aconselhada a terapia com anti-hipertensivo oral (Malachias, et al., 2016). 
A seleção do anti-hipertensivo a ser utilizado depende da segurança e eficácia que o fármaco apresenta para a mãe e o feto, visando à administração de doses mínimas e à escolha de medicamentos que possuem apenas um fármaco em sua formulação, com o intuito de conter prováveis efeitos colaterais. Portanto, são adotados fármacos que possuem mais informações registradas na literatura (Olson-Chen \& Seligman, 2016). A Tabela 4 apresenta os fármacos mais utilizados no tratamento da hipertensão arterial na gestação.

Tabela 4- Os fármacos mais utilizados no tratamento da hipertensão arterial na gestação.

\begin{tabular}{l|c|c}
\hline CLASSE & \multicolumn{2}{c}{ FÁRMACO } \\
Alfa-agonistas & Metildopa & Categoria B \\
\hline Betabloqueadores & Pindolol & Categoria B \\
\hline $\begin{array}{l}\text { Bloqueadores dos canais } \\
\text { de cálcio tipo 2 }\end{array}$ & Nifedipina & Categoria C \\
\hline Vasodilatadores & Cloridrato de Hidralazina & Categoria C \\
\hline
\end{tabular}

Fonte: Adaptado de Siqueira, et al., 2011.

Os medicamentos anti-hipertensivos de primeira escolha durante o tratamento da Hipertensão Gestacional (HG) abrangem a metildopa, que é o fármaco anti-hipertensivo mais utilizado neste quadro, e o único cujas pesquisas avaliaram o desenvolvimento em logo prazo de crianças submetidas ao seu efeito intrauterino. Assim como a Nifedipina, que, quando comparada com a metildopa, apresenta efeito anti-hipertensivo similar, mas não prolonga o tempo de gestação ou melhora o prognóstico fetal, sendo usada em sua forma de liberação lenta no tratamento contínuo da hipertensão na gravidez e da emergência hipertensiva (Santos \& Capobianco, 2019).

Entre os betabloqueadores, o pindolol é considerado um fármaco de primeira linha para a monoterapia da hipertensão na gravidez, demonstrando ser o mais seguro desta classe para o feto, pois permeia a placenta, mas não gera restrição de crescimento e alteração da função cardíaca fetal (Blais, et al., 2018; Butalia, et al., 2018). E quando comparado ao alfa agonista, metildopa, apresenta resultado terapêutico mais eficaz (Magee, et al., 2014). Já a hidralazina é um potente vasodilatador que diminui a resistência vascular periférica, promovendo o relaxamento da musculatura lisa das artérias. Por via oral, é considerada de 
segunda ou terceira opção, porém, na crise hipertensiva, é o anti-hipertensivo mais empregado, sendo administrado por via endovenosa (Silva, et al., 2015).

\subsection{Diabetes mellitus gestacional}

Na gravidez, a glicose materna corresponde à principal fonte de energia para o feto, consistindo em um período que promove grandes transformações no metabolismo energético da mulher, o qual possui duas fases distintas. Dessa forma, os hormônios estrogênio e progesterona, no primeiro trimestre, agem nas células do pâncreas, amplificando a produção de insulina, resultando na redução da glicemia e aumento da reserva de glicogênio e de gordura (Barros, et al., 2019).

Já no segundo e terceiro trimestres, devido ao aumento na produção de hormônios hiperglicemiantes, em suma, o lactogênio placentário humano, ocorre o aumento da resistência periférica à insulina, com consequente variação nos níveis de glicose no sangue em relação aos parâmetros normais e diminuição da reserva de glicogênio e gordura (Massucatti; Pereira \& Maioli, 2012). Estas variações metabólicas, ao decorrer da gestação, oportunizam o surgimento da Diabetes Mellitus Gestacional (DMG), que se traduz como intolerância à glicose, a qual inicia ou tem o primeiro diagnóstico durante a gestação, podendo ou não perdurar após o parto (Silva, et al., 2019).

Dentre os fatores de risco que viabilizam o aparecimento da Diabetes Mellitus Gestacional, destacam-se o sobrepeso materno, que está relacionado ao ganho de peso excessivo durante a gestação, o histórico familiar de parentes de primeiro grau, a existência de hipertensão ou pré-eclâmpsia na gravidez atual, bem como o aumento da idade materna, que apresenta uma relação diretamente proporcional à incidência deste problema metabólico (Fernandes \& Bezerra, 2020).

Com respeito à prevenção de problemas provocados por esse distúrbio metabólico durante a gestação, constata-se a relevância do controle glicêmico associado à dieta, consistindo na escolha preliminar de tratamento, assim como da atividade física, cujo objetivo primordial é a redução da intolerância à glicose, e da terapia medicamentosa (Neta, et al., 2014). A Tabela 5 apresenta os fármacos mais utilizados no tratamento do diabetes mellitus gestacional. 
(CC BY 4.0) | ISSN 2525-3409 | DOI: http://dx.doi.org/10.33448/rsd-v9i7.4626

Tabela 5- Os fármacos mais utilizados no tratamento do diabetes mellitus gestacional.

\begin{tabular}{l|c|c}
\hline \multicolumn{1}{c}{ CLASSE } & FÁRMACO & $\begin{array}{c}\text { CLASSIFICAÇÃO } \\
\text { DE RISCO }\end{array}$ \\
\hline Insulinas & Regular e NPH & Categoria B \\
\hline Biguanidas & Metformina & Categoria B \\
\hline Sulfonilureias & Glibenclamida & Categoria C \\
\hline
\end{tabular}

Fonte: Adaptado de Febrasgo, 2011.

O tratamento usualmente é iniciado com a implantação de um plano alimentar e exercício físico. Não havendo normalização dos níveis glicêmicos em uma a duas semanas após a adoção dessas medidas, é recomendado iniciar o tratamento farmacológico, preferivelmente com insulina, pois esta não atravessa a barreira placentária, e todos os tipos podem ser utilizados, mas a insulina recombinante humana tem menos atividade imunogênica e deve ser preferida (Miranda, et al., 2017).

Contudo, a metformina e a glibenclamida são exemplos de antidiabéticos orais que também podem ser utilizados na gravidez. Algumas pequenas pesquisas clínicas observaram que a metformina se mostra eficaz tanto quanto a insulina para tratamento da diabetes gestacional sem acrescer o risco de problemas neonatais ou maternos (Cesta, et al., 2019). A metformina permeia a placenta, porém não foram comprovados efeitos teratogênicos, há evidência científica da segurança e eficácia do seu uso durante a gravidez. Já a passagem transplacentar da glibenclamida ainda não está bem evidenciada, não sendo comprovado qualquer efeito teratogênico (Febrasgo, 2011).

Apesar de não haver relatos de efeitos adversos com os fármacos citados, é preciso cuidado na indicação dessas medicações, sendo que a insulina continua sendo a primeira escolha para o tratamento do DMG (Arshad; Karim \& Hason, 2014). 


\subsection{Medicamentos antieméticos}

Alguns fatores hormonais e mecânicos provenientes da gestação promovem uma série de modificações em vários aparelhos e sistemas do corpo materno, com destaque ao aparelho digestivo, pois aproximadamente 50 a $80 \%$ das gestantes relatam sofrer com náuseas e vômitos. Estes podem se manifestar em qualquer horário do dia, ocorrendo mais comumente pela manhã, ao levantar-se, e desaparecem em torno da $16^{\mathrm{a}}$ a $20^{\mathrm{a}}$ semana. Contudo, em torno de $5 \%$ dos casos, verifica-se a evolução para um estado grave, denominado de hiperemese gravídica, gerando desidratação, desnutrição, desequilíbrios hidroeletrolíticos e ácidos-básicos (Oliveira, et al., 2010).

A causa que desencadeia as náuseas e vômitos ainda não foi esclarecida, porém, julgase que tenha ligação ao aumento da gonadotrofina coriônica humana e estrogênio, além da diminuição da acidez do estômago, do tônus e da motilidade do trato gastrintestinal (Aguiar, et al., 2013).

Com relação à terapia não farmacológica, inclui o fracionamento alimentar, ou seja, a realização de seis ou mais refeições por dia, o consumo de alimentos sólidos ao acordar e gengibre, evitar a ingestão de frituras, gorduras, alimentos condimentados e com forte odor, bem como de líquidos durante as refeições. No tocante ao tratamento farmacológico, a utilização dos antieméticos durante a gravidez deve ser realizada com cuidado, não devendo ser usados antes da 12a a $14^{\mathrm{a}}$ semanas (S/SUBPAV/SAP, 2013). A Tabela 6 apresenta os fármacos mais utilizados no tratamento de náuseas e vômitos durante a gravidez.

Tabela 6- Os fármacos mais utilizados no tratamento de náuseas e vômitos.

\begin{tabular}{l|c|c}
\multicolumn{1}{c}{ CLASSE } & FÁRMACO & $\begin{array}{c}\text { CLASSIFICAÇÃO } \\
\text { DE RISCO }\end{array}$ \\
\hline $\begin{array}{l}\text { Antagonistas dos receptores } \\
\text { dopamínicos D }\end{array}$ & $\begin{array}{c}\text { Cloridrato de } \\
\text { Metoclopramida }\end{array}$ & Categoria B \\
\hline $\begin{array}{l}\text { Antagonistas dos receptores } \\
\text { histamínicos H1 / Vitamina }\end{array}$ & $\begin{array}{c}\text { Dimenidrinato + Cloridrato } \\
\text { de Piridoxina }\end{array}$ & Categoria B \\
\hline $\begin{array}{l}\text { Antagonistas dos receptores } \\
\text { histamínicos H1 }\end{array}$ & Cloridrato de Prometazina & Categoria C \\
\hline
\end{tabular}

Fonte: Adaptado de S/SUBPAV/SAP, 2013. 
O uso da metoclopramida durante a gestação mostra-se seguro, pois, ainda que atravesse a placenta, não existem efeitos teratogênicos relatados em animais ou humanos e apresenta menos efeitos adversos em relação à prometazina (Gilboa, et al., 2014). Já a piridoxina, também denominada vitamina B6, é solúvel em água e consiste em uma coenzima essencial na via do metabolismo do folato, tendo seu uso regular considerado eficiente na redução da frequência dos vômitos e intensidade das náuseas (Babaei \& Mahboube, 2014).

\subsection{Automedicação na gestação}

$\mathrm{O}$ ato de utilizar medicamentos isentos de prescrição e que se encontram seguros e eficazes para tratar sintomas autodiagnosticados, sem o aconselhamento e/ou acompanhamento de um profissional de saúde qualificado é intitulado de automedicação, que, segundo a Organização Mundial de Saúde (OMS), quando realizada de forma responsável, traz somente vantagens ao indivíduo e ao sistema de saúde em geral, reduzindo o tempo de permanência dos sintomas e permitindo que os profissionais de saúde direcionem-se aos problemas clínicos mais graves, amenizando a sobrecarga dos serviços (Arrais, et al., 2016).

Porém, diversos estudos consideram a automedicação um motivo de grande preocupação, principalmente quando realizada por gestantes, devido aos potenciais riscos fetais promovidos por algumas substâncias (Ribeiro, 2013).

Apesar dos riscos, a pesquisa de Santos et al. (2018) mostra que automedicação na gravidez se caracteriza como um fenômeno frequente, pois $33,75 \%$ das gestantes entrevistadas citaram a prática da automedicação, bem como perigoso, uma vez que $11,1 \%$ destas gestantes relataram mal-estar após a utilização de alguns medicamentos, dentre os quais, alguns possuem informe de contraindicação na bula registrada pela ANVISA. Além disso, estudos demostram que a maioria das mulheres grávidas estão em uso de um ou mais fármacos no período gestacional, com taxa variando entre 50 a $80 \%$ e próximo a $100 \%$, para fármacos prescritos e sem prescrição, respectivamente aumentando as chances de complicações para o mãe e feto (Stock \& Norman, 2019).

\subsection{Importância da atenção farmacêutica}

A atenção farmacêutica é introduzida no ciclo da assistência farmacêutica através da dispensação e utilização dos medicamentos, consistindo em um serviço prestado pelo farmacêutico ao paciente através do contato direto, com o intuito de promover uma 
farmacoterapia racional e a conquista de resultados estabelecidos e mensuráveis, visando o aumento da qualidade de vida (Coradi, 2012).

A atenção farmacêutica, seja no setor público ou privado, deve ofertar todo o suporte possível às gestantes, viabilizando o uso responsável de medicamentos em uma fase classificada como delicada e complexa, uma vez que o farmacêutico se revela como o profissional mais fácil acesso à gestante, sendo, em muitos casos, a sua fonte primária de conhecimento acerca do uso de medicamentos (Germano, et al., 2016).

Neste cenário, o profissional farmacêutico é de fundamental importância para o tratamento da hipertensão arterial na gestação e diabetes mellitus gestacional tanto pela capacidade de analisar o risco promovido pelo uso dos fármacos, verificando se há interação com outros medicamentos ou alimentos utilizados pela paciente, favorecendo, assim, uma farmacoterapia racional e segura, bem como pelo conhecimento de medidas nãofarmacológicos que consistem em ações que auxiliam o tratamento e evitam complicações no binômio mãe-feto, e pela disponibilidade de acompanhamento regular da pressão arterial através da aferição da mesma e da glicemia capilar, possibilitando verificar se a terapia alcançou o resultado desejado, obtendo aptidão necessária para a sugestão de alguma intervenção terapêutica (Rodrigues, et al., 2018; Rolim, et al., 2016).

O auxílio no processo de adesão terapêutica também faz parte das condutas executadas através da atenção farmacêutica, pois o profissional farmacêutico como dispensador de medicamentos tem um papel importante na observação dos problemas que dificultam a adesão terapêutica e em conjunto com o paciente estabelecer estratégias que os solucionem, sempre reforçando a importância da adesão principalmente em doenças crônicas e no período gestacional (Belhabib, et al., 2018).

\section{Considerações Finais}

De acordo com os resultados apresentados, foi possível concluir que todos os tratamentos farmacológicos realizados durante a gravidez devem ser pontualmente orientados devido aos ricos existentes na automedicação e executados com competência, utilizando medicamentos seguros e com doses exatas por se tratar de dois organismos que respondem de forma distinta às substâncias.

O profissional farmacêutico denota um papel importantíssimo no alcance desses requisitos, por possuir o conhecimento científico necessário para promover indicações e intervenções terapêuticas, respectivamente seguras e necessárias, bem como a capacidade de 
alcance dessas pacientes através do serviço de atenção farmacêutica, que possibilita o contato direto e contínuo.

Principalmente nos cenários de HG e DMG, o valor da atenção farmacêutica é revelado, pois aqueles exibem ampla relevância na saúde pública brasileira em consequência da prevalência que apresentam, como também das complicações e riscos materno-fetais que podem desenvolver, necessitando do monitoramento dos seus respectivos parâmetros de referência e da adesão terapêutica.

\section{Referências}

Aguiar, R. S. et al. (2013). Orientações de enfermagem nas adaptações fisiológicas da gestação. Cogitare Enfermagem, 18(3), 527-531.

Anvisa. Agência Nacional de Vigilância Sanitária. Resolução da diretoria colegiada- RDC Nº 60, de 17 de dezembro de 2010. Disponível em:< http://bvsms.saude.gov.br/bvs/saudelegis/anvisa/2010/res0060_17_12_2010.html> Acessado em: 03 abr. 2020.

Arrais, P. S. D. et al. (2016). Prevalência da automedicação no Brasil e fatores associados. Revista de Saúde Pública, 50, 1-13.

Arshad, R., Karim, N. \& Hason, J. A. (2014). Effects of insulin on placental, fetal and maternal outcomes in gestacional diabetes mellitus. Pakistan Journal of Medical Sciences, $30(2), 240-244$.

Babaei, A. H. \& Mahboube, H. F. (2014). A randomized comparison of vitamin B6 and dimenhydrinate in the treatment of nausea and vomiting in early pregnancy. Iranian Journal of Nursing and Midwifery Research, 19(2), 199- 202.

Barros, G. M. et al. (2019). Idade como fator de risco para diabetes mellitus gestacional. Ciência, Cuidado e Saúde. 18(2), 1-8.

Belhabib, G. et al. (2018). Evaluation of factors for therapeutic adherence in diabetic patients. Le Pharmacien Hospitalier et Clinicien. 53, 159-167. 
Blais, L. et al. (2018). Long-Acting b2-Agonists and Risk of Hypertensive Disorders of Pregnancy: A Cohort Study. Journal Allergy Clin Immunol pract. 6(2), 555-561.

Brasil. Sociedade Brasileira de Cardiologia. VI Diretrizes brasileiras de hipertensão. Arquivos Brasileiros de Cardiologia, Rio de Janeiro, 2010. 51 p.

Butalia, S. et al. (2018). Hypertension Canada's 2018 Guidelines for the Management of Hypertension in Pregnancy. Canadian Journal of Cardiology. 34(5), 526-531.

Cesta, C. E. et al. (2019). Antidiabetic medication use during pregnancy: an international utilization study. BMJ Open Diabetes Research \& Care, 7, 1-12.

Coradi, A. E. P. (2012). A importância do farmacêutico no ciclo da assistência farmacêutica. Arquivos Brasileiros de Ciências da Saúde, 37(2), 62-64.

Febrasgo. Federação Brasileira das Associações de Ginecologia e Obstetrícia. Manual de Gestação de Alto Risco. Rio de Janeiro: FEBRASGO, 2011.

Fernandes, C. N \& Bezerra, M. M. M. (2020) O diabetes mellitus gestacional: causa e tratamento. Revista Multidisciplinar e de Psicologia, 14(49),127-139.

Ferreira, S. S. et al. (2017). Ácido acetilsalicílico na prevenção da pré-eclâmpsia: uma revisão baseada na evidência. Revista Portuguesa de Medicina Geral e Familiar, 33, 118-132.

Food Drug and Administration. FDA/CDER SBIA Chronicles. Drugs in Pregnancy and Lactation: Improved Benefit-Risk Information. Acesso em 22 de maio de 2020. Disponível em: < https://www.fda.gov/files/drugs/published/\%22Drugs-in-Pregnancy-and-Lactation-Improved-Benefit-Risk-Information\%22-January-22--2015-Issue.pdf>

Germano, M. C. M. et al. Gestantes com eclâmpsia no sertão cearense: terapia medicamentosa e o uso racional. In: ANAIS DA AMOSTRA CIENTÍFICA DA FARMÁCIA, 10., 2016, Quixadá. Anais... Quixadá: Centro Universitário Católica de Quixadá, 2016. 
Gilboa, S. M. et al. (2014) Antihistamines and birth defects: a systematic review of the literature. Expert Opinion on Drug Safety, 13(12),1667-98.

Kahhale, S; Francisco, R. P. V \& Zugaib, M. (2018). Pré-Eclampsia. Revista de Medicina, 97(2), 226-234.

Magee, L.A. et al., (2014). Diagnosis, evaluation, and management of the hypertensivedisorders of pregnancy. Pregnancy Hypertension: An InternationalJournal of Women's Cardiovascular Health, 4,105-145.

Malachias, M. V. B. et al. (2016). $7^{\text {a }}$ Diretriz Brasileira de Hipertensão Arterial: Capítulo 9 Hipertensão Arterial na gestação. Arquivos Brasileiros de Cardiologia, 107(3).

Massucatti, L. A.; Pereira, R. A \& Maioli, T. U. (2012). Prevalência de diabetes gestacional em unidades de saúde básica. Revista de Enfermagem e Atenção à Saúde, 1(1), 70-79.

Melo, W. F. et al. (2015). A hipertensão gestacional e o risco de pré-eclâmpsia: revisão bibliográfica. Revista Brasileira de Educação e Saúde, 5(3), 07-11.

Menezes, M. S. S. et al. (2014). Uso de medicamentos por gestantes atendidas no Hospital da Polícia Militar - Mossoró/RN. Revista Brasileira de Farmácia, 95(1), 512-529.

Miranda, A. et al. (2017). Diabetes gestacional: avaliação dos desfechos maternos, fetais e neonatais. Revista Portuguesa de Endocrinologia, Diabetes e Metabolismo, 12, (1), 36-44.

Moro, A. \& Invernizze, N. (2017). A tragédia da talidomida: a luta pelos direitos das vítimas e por melhor regulação de medicamentos. História, Ciências, Saúde-Manguinhos, 24(3), 603622.

Moura, E. R. F. (2010). Fatores de risco para síndrome hipertensiva específica da gestação entre mulheres hospitalizadas com pré-eclâmpsia. Cogitare Enfermagem, 15(2), 250-255. 
Nascimento, A. M. et al. (2016). Avaliação do uso de medicamentos por gestantes em unidades básicas de saúde de Rondonópolis, Mato Grosso. Revista Eletrônica Gestão \& Saúde, 7(1), 96-112.

Nascimento, R.C.C.Z.M. et al. (2017). Polifarmácia: uma realidade na atenção primária do Sistema Único de Saúde. Revista de Saúde Pública, 51(2),1-12.

Neta, F. A. V. et al. (2014). Avaliação do perfil e dos cuidados no pré-natal de mulheres com diabetes mellitus gestacional. Revista Rene, 15(5),823-831.

Oliveira, G. K. S. et al. (2010). Intervenções de enfermagem nas adaptações fisiológicas da gestação. VAREDAS - Revista Eletrônica de Ciências, 3(1),58-67.

Olson-chen, C. \& Seligman, N. S. (2016). Hypertensive Emergencies in Pregnancy. Critical care clinics, 32(1),29-41.

Prado, I. F. et al. (2017). Hipertensão arterial durante a gravidez. Revista Portuguesa de Ciências do Desporto, 17(3), 65-77.

Ribeiro, A. S. et al. (2013). Risco potencial do uso de medicamentos durante a gravidez e a lactação. Infarma - Ciências Farmacêuticas, 25(1), 62-67.

Rodrigues, A. S. et al. (2018). Assistência farmacêutica no âmbito de cuidados a gestantes com hipertensão arterial. Revista Científica FAEMA, 9, 540-546.

Rolim, C. E. et al. (2016). A importância da atenção farmacêutica e a diabetes mellitus tipo 2. INTESA - Informativo Técnico do Semiárido, 10(2), 92-104.

S/SUBPAV/SAP. Guia de referência rápida - Atenção ao pré-natal: rotinas para gestantes de baixo risco. Rio de Janeiro, Superintendência de Atenção Primária (S/SUBPAV/SAP), 2013. $1^{\mathrm{o}}$ edição.

Santos, M. J. \& Capobianco, M. P. (2019). Hipertensão gestacional. Revista União das Faculdades dos Grandes Lagos, 1(1), 1-14. 
Santos, S. L. F. et al. (2018). Automedicação em gestantes de alto risco: foco em atenção farmacêutica. Journal of Health Sciences, 20(1), 50-54.

Silva, B. Y. C. et al. (2011). Inter-relações entre consumo de cálcio e pré-eclâmpsia. Revista Brasileira de Nutrição Clínica, 26 (4),264-269.

Silva, R. R. et al. (2019). Desfechos materno-fetais de gestantes com e sem diabetes mellitus gestacional. Arquivos Catarinenses de Medicina, 48(3), 79-92.

Silva, S. D. et al. (2015). Quando introduzir o tratamento farmacológico na pré-eclâmpsia. Revista HUPE, 14(2), 87-93.

Siqueira, F. et al. (2011). Medicamentos anti-hipertensivos na gestação e puerpério. Revista Comunicação em Ciências da Saúde, 22(1), 55-68.

Stock, J.E \& Norman, J.E (2019). Medicines in pregnancy [version 1; peer review: 3 approved]. F1000 Research, 8(911), 1-8.

Van Gelder, M. M. H. J.; Jong-Van den Berg, L. T. W \& Roeleveld, N. (2014). Drugs associated with teratogenic mechanisms. Part II: a literature review of the evidence on human risks. Human Reproduction, 29(1),168-183.

Vettore, M. V. et al. (2011). Cuidados pré-natais e avaliação do manejo da hipertensão arterial em gestantes do SUS no Município do Rio de Janeiro, Brasil. Caderno de Saúde Pública,27(5), 1021- 1034.

\section{Porcentagem de contribuição de cada autor no manuscrito}

Damiris de Carvalho Vieira Guedes - 50\%

Samara Alves Brito - 10\%

Danielle Rocha Silva - $40 \%$ 